Document downloaded from:

http://hdl.handle.net/10251/83358

This paper must be cited as:

Rius Mercado, M.; Bolea Boluda, M.; Mora Almerich, J.; Capmany Francoy, J. (2016). HighOrder UWB Pulses Generation Adaptable to Bi-Phase Modulation. IEEE Photonics Technology Letters. 28(21):2371-2374. doi:10.1109/LPT.2016.2594154.

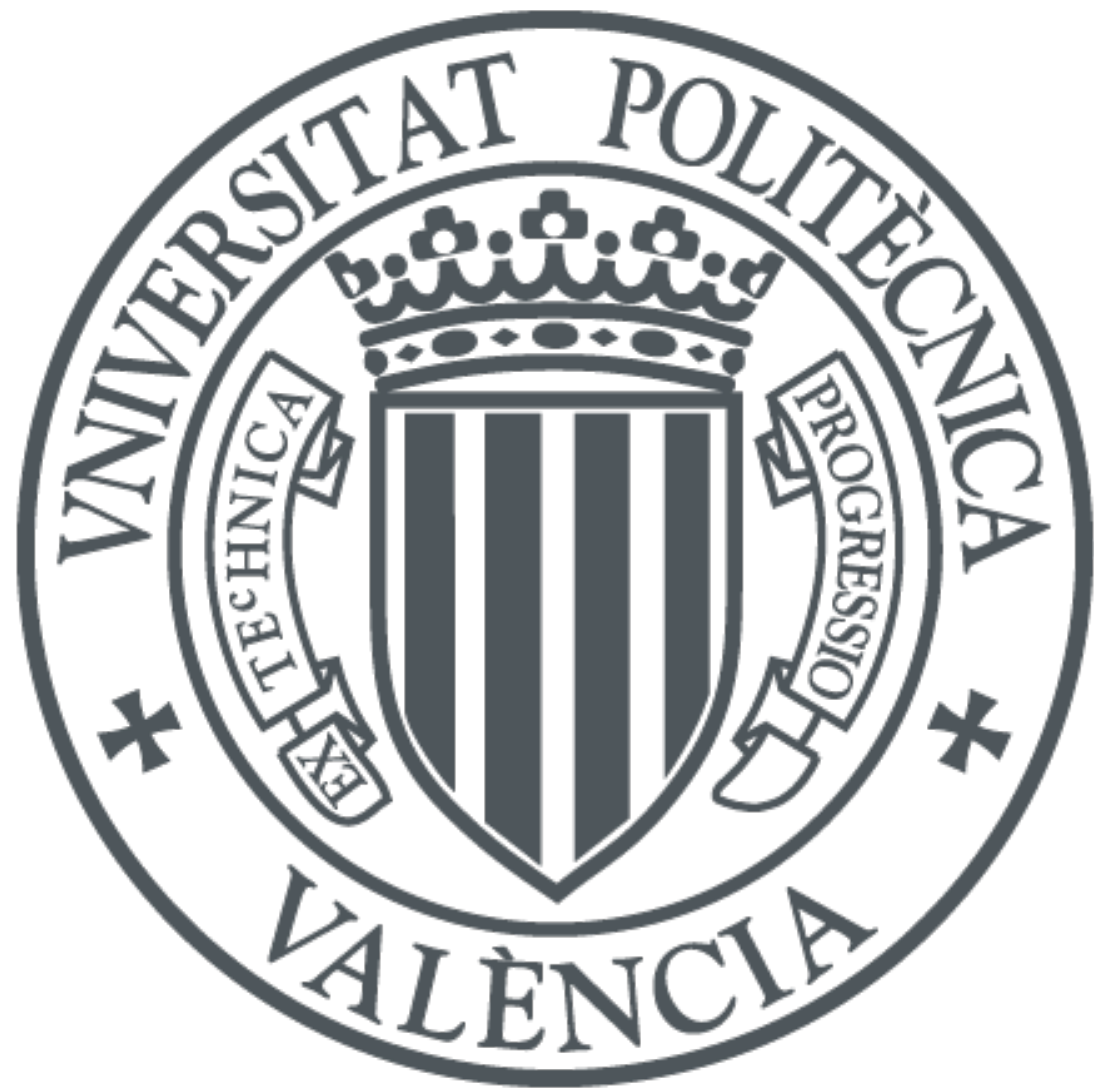

The final publication is available at

http://dx.doi.org/10.1109/LPT.2016.2594154

Copyright Institute of Electrical and Electronics Engineers (IEEE)

Additional Information

(c) 2016 IEEE. Personal use of this material is permitted. Permission from IEEE must be obtained for all other users, including reprinting/ republishing this material for advertising or promotional purposes, creating new collective works for resale or redistribution to servers or lists, or reuse of any copyrighted components of this work in other works. 


\title{
High-order UWB Pulses Generation adaptable to Bi-Phase Modulation
}

\author{
Manuel Rius, Mario Bolea, José Mora and José Capmany, Fellow, IEEE.
}

\begin{abstract}
In this paper, we present a high-order ImpulseRadio Ultra-Wideband (IR-UWB) pulses generator based on the processing of incoherent optical signals by a dispersive element. The full waveform reconfigurability is achieved by the control of the optical signal power spectral density profile. Operation principle is experimentally shown by the generation of classical UWB pulses: monocycle and doublet. High-order UWB pulses generation is experimentally demonstrated by the generation of triplet and quadruplet. Also, the proper adjustment of the optical signal power distribution permits to reduce the baseband content of the synthesized waveform which is critical in UWB environments to achieve spectrally efficient solutions. Moreover, system flexibility allows the adaptation to a Bi-Phase Modulation scheme. This feature has been proved by the generation of the inverted version of the UWB pulses achieved in the manuscript.
\end{abstract}

Index Terms - Impulse-Radio Ultra-Wideband, wireless communication, optical source, dispersive element, high-order pulses, Bi-Phase Modulation format.

\section{INTRODUCTION}

$\mathrm{T}$ The Impulse-Radio Ultra-wideband (IR-UWB) has been considered to be a promising technology for short-reach wireless communications because it can potentially allow new services to coexist with the existing narrowband wireless services with minimal interference. UWB characterizes transmission system with instantaneous spectral occupancy in excess of $500 \mathrm{MHz}$ or a fractional bandwidth of more than 20 \%. As information-bearing pulses with ultra-short duration have an UWB spectral occupancy, UWB radio transmissions come with unique advantages such as: capability to penetrate through obstacles, immunity to multipath fading, potential for very high data rates along with a commensurate increase in user capacity and potentially small sized and low processing power. For wireless communications, the US Federal Communications Commission (FCC) defines the use without license of the spectrum from 3.1 to $10.6 \mathrm{GHz}$ with a restriction in power spectral density of $-41.3 \mathrm{dBm} / \mathrm{MHz}[1,2]$.

Wireless UWB-signals range is kept to less than tens of meters by maintaining a low radiation power density. In this point, UWB-over-fibre serves as an effective solution to

Manuscript received July 1st, 2016. The research leading to these results has received funding from the national project TEC2014-60378-C2-1-R funded by the Ministerio de Ciencia y Tecnología and the regional project PROMETEO FASE II/2013/012 funded by the Generalitat Valenciana.

M. Rius, M. Bolea, J. Mora and J. Capmany are with the ITEAM Research Institute, Universidad Politécnica de Valencia, Valencia, Spain (e-mail: jmalmer@iteam.upv.es). distribute IR-UWB signals. For such optical distribution, it is more cost-effective to implement the generation and distribution of IR-UWB in the optical domain and perform the conversion to the RF wireless domain at the remote antenna units, thus avoiding the need for multiple electrical-to-opticalto-electrical $(\mathrm{E} / \mathrm{O} / \mathrm{E})$ conversions in the generation and distribution of IR-UWB [3]. IR-UWB photonic generation and free space transmission for wireless communications have been experimentally demonstrated [4], highlighting the potential of IR-UWB photonic generation in RoF systems.

Recently, a huge number of approaches have been reported to generate UWB signals. We can found schemes to generate classical UWB pulses, i.e. monocycles and doublets, based on phase modulation to intensity modulation (PM-IM) conversion by using an optical frequency discriminator implemented by optical filters [5] or silicon microring modulators [6]. Moreover, microwave photonic filters have been also proposed for UWB signals generation implementing negative taps based on the nonlinear amplification process in SOA [7]. We can also find schemes based on optical spectral shaping and dispersion-induced frequency-to-time mapping [8].

The most problems of UWB transmission technology for wireless communications are around low frequencies. The FCC establishes the higher power spectral restriction around the GPS band (0.96-1.61 GHz) [1, 2]. The last times, highorder pulses generation have been demonstrated to achieve a more efficient use of the UWB available spectrum [9]. In this way, the coverage area of the UWB radio signals can be increased. In the literature, we can found approaches based on microwave photonic filtering with different lasers and dispersive elements [9] and cross-phase modulation in a SOAMZI [10]. Also, high order UWB pulses can be achieved by the combination of classical monocycle [11] and doublet [12] waveforms each one obtained by PM-IM conversion.

Apart of an efficient use of the FCC mask, the adaptability to UWB modulation formats is another field of interest in which UWB communication is focused recently [9]. Nevertheless, the most of schemes reported can only implement on-off keying (OOK) modulation. The limitation of the OOK modulation of UWB is the low immunity to multipath effect in which echoes of the original pulses make difficult to determine the absence of pulse. However, Bi-Phase modulation (BPM) can overcome this limitation and presents an improvement of the signal-to-noise ratio (SNR) of $3 \mathrm{~dB}$. Thus, UWB BPM has attracted great interest in the photonic generation of UWB pulses [7, 9]. 


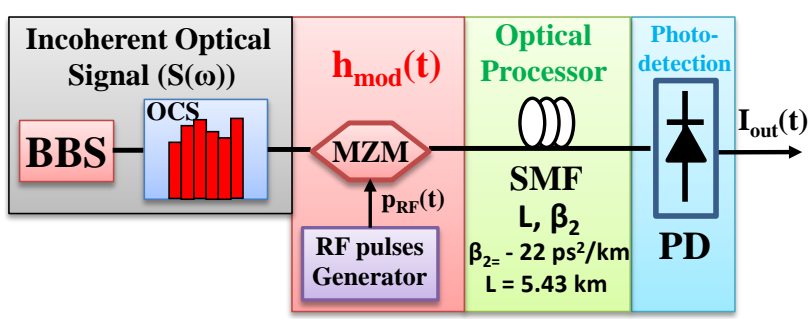

Fig. 1. Experimental scheme of the proposed IR-UWB pulse generator.

In this paper, we propose and experimentally demonstrate a high-order IR-UWB generator which can implement UWB BPM modulation scheme. The system proposed is based on the processing of incoherent signals by means of dispersive elements. Positive and negative pulses are easily achieved through the corresponding shaping of the incoherent optical signal. The amplitude and polarity of these optical pulses can be controlled independently. Moreover, the propagation through a dispersive element introduces the corresponding difference time delay between pulses in order to generate different UWB waveforms. High-order IR-UWB pulses generation is experimentally demonstrated by obtaining different waveforms such as: monocycle, doublet, triplet and quadruplet. Furthermore, through the control over the optical source power profile inverted waveforms has been synthesized which permits to adapt the system to UWB Bi-Phase Modulation format. All the experimental results, both optical power distribution and generated waveform with its corresponding electrical power spectrum are single shot measurements, i.e. no averaging is applied [13].

\section{SYSTEM DESCRIPTION}

The experimental scheme of the system proposed for generating high order UWB pulses is shown in Fig. 1. The operation principle is based on the processing of an incoherent optical signal by means of a dispersive element. From the theoretical analysis performed in [14], when second order dispersion is neglected $\left(\varphi_{3}=0\right)$, this approach can be considered as an incoherent frequency-to-time mapping. We consider an incoherent optical signal with a power spectral density $S(\omega)$, centered at $\omega_{0}$, such that the optical field of the light describes a stationary random process. Each optical frequency is modulated by means of a modulator with impulse response $h \bmod (t)$. Then, the modulated signal is launched into an optical processor which is determined by the first-order dispersion $\left(\varphi_{2}=\beta_{2} L\right)$ evaluated at $\omega_{0}$. In this way, the electrical signal obtained $\left(\mathrm{I}_{\text {out }}(\mathrm{t})\right)$ after the photodetection process is given by [14]:

$$
I_{\text {out }}(t)=\frac{I_{o}}{2 \pi \varphi_{2}} S\left(\omega=t / \varphi_{2}\right) \otimes\left|h_{\text {mod }}\left(\omega_{0}, t\right)\right|^{2}
$$

where the first term corresponds to a time-domain scaled version of the power spectrum $S(\omega)$ and the second term $\mathrm{h}_{\bmod }\left(\omega_{0}, \mathrm{t}\right)$ represents the output pulse when a monochromatic source is considered. Note that, apart from the frequency-totime mapping process, the generated waveform will be also affected by hmod(t).

First of all, the incoherent optical signal is generated using an optical source composed by a Broadband Source (BBS) and an Optical Channel Selector (OCS) (Peleton QTM100C). The BBS has a total optical bandwidth of $80 \mathrm{~nm}$ and the OCS is centered at $\lambda_{0}=1546.92 \mathrm{~nm}$ with 48 channels of $0.8 \mathrm{~nm}$ width. Each channel attenuation can be controlled independently up to a maximum of $20 \mathrm{~dB}$ when the channel is blocked. In this way, the control of those channels permits to synthesize different optical power distributions $(S(\omega))$. Next, the shaped optical signal is modulated by a RF pulse $\left(p_{R F}(t)\right)$ using a Mach-Zehnder Modulator (MZM) (Photline MX-LN40-PD). This RF pulse is obtained from a RF pulse generator (Anritsu MP1800A) which is configured at a bit rate of 12.5 $\mathrm{Gb} / \mathrm{s}$ with a pattern of one " 1 " and thirty-one " 0 ". Therefore, the repetition rate is close to $390.65 \mathrm{MHz}\left(1 / \mathrm{T}_{\mathrm{w}}\right)$ and the pulse width is 80 ps. The modulated optical signal is propagated through a standard single mode fibre link (SSMF-28) with a length (L) of $5.43 \mathrm{Km}$ and a first order dispersion parameter $\beta_{2}=-22 \mathrm{ps}^{2} / \mathrm{Km}$. Finally, the resulting waveform $\left(\mathrm{I}_{\text {out }}(\mathrm{t})\right)$ is obtained after the corresponding photodetection (PD) process.
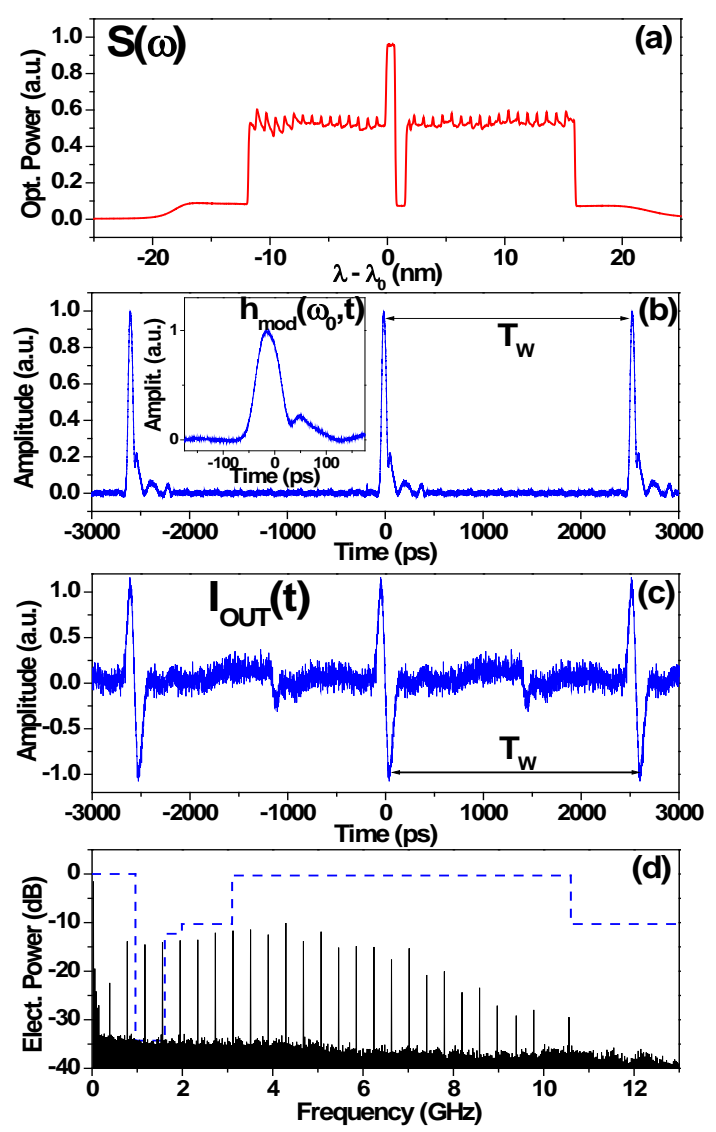

Fig. 2. (a) Power spectral density of the incoherent optical signal respect to the central wavelength. (b) Output electrical pulses for a monochromatic source, inset $h_{\bmod }\left(\omega_{0}, t\right)$. (c) Generated monocycle pulse and (d) corresponding electrical power spectrum. FCC mask added in dashed line. 

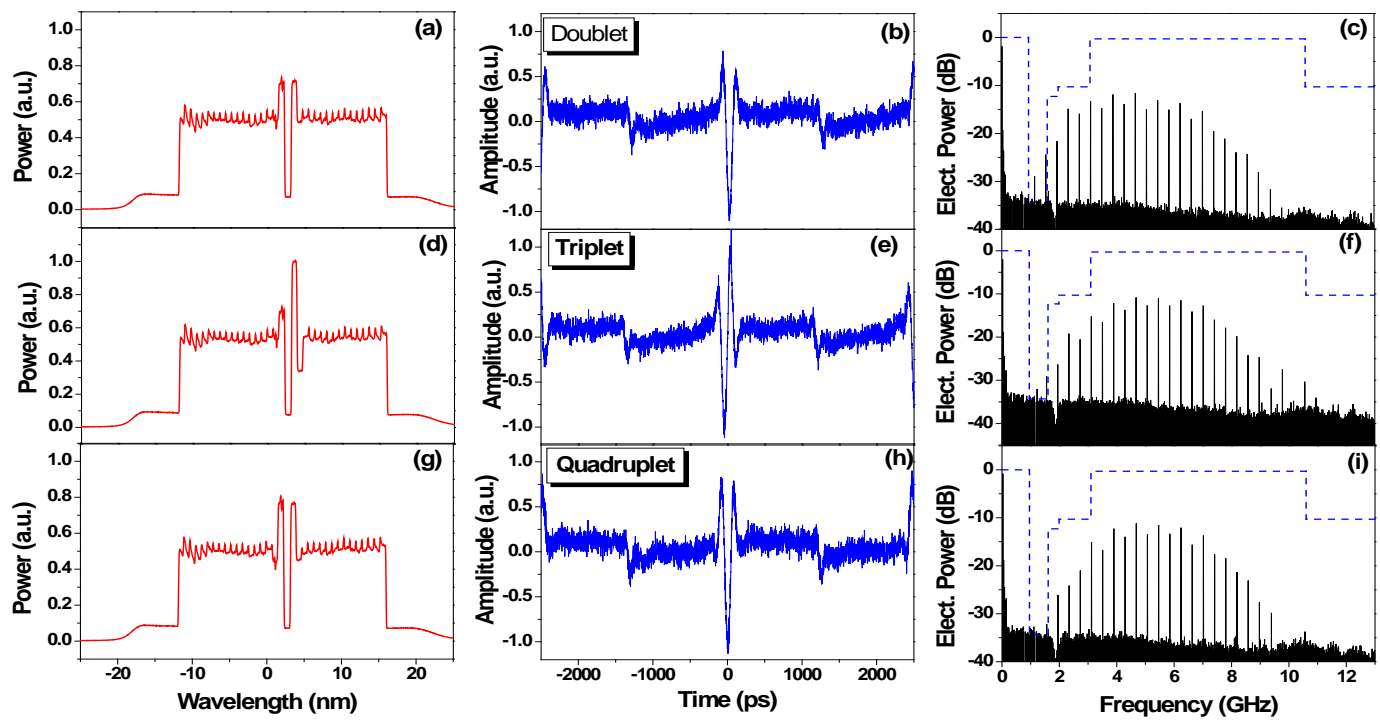

Fig. 3. Experimental results of the optical source power spectral density, generated waveforms electrical power spectrum for doublet (a), (b), (c); triplet (d), (e), (f); and quadruplet (g), (h), (i), respectively. FCC mask has been added in blue dashed line.

The performance of the proposed system is analyzed by generating a monocycle IR-UWB waveform. By means of the OCS, the incoherent optical signal spectrum is shaped to a power spectral density distribution as shown Fig. 2(a), S( $\omega)$. In this case, an optical bandwidth of $28 \mathrm{~nm}$, corresponding to 35 active channels of the OCS, has been performed. Moreover, all active channels have been attenuated in order to obtain a uniform power distribution at the half of the maximum optical power available. Nevertheless, the active channel at $1546.92 \mathrm{~nm}$ has been maintained with the maximum power and the following active channel at 1547.72 $\mathrm{nm}$ has been totally attenuated, i.e., the channel has been blocked. Taking into account that the uniform profile at the half of the maximum optical power available corresponds to a continuous level, we can define the equivalent amplitude levels $[1,-1]$ for describing the incoherent signal power profile. Fig 2(b) shows the output electrical signal when a monochromatic source is considered for the RF pulse generator configuration previously mentioned. Note that one of this pulses (inset Fig. 2(b)) corresponds to $h_{\bmod }\left(\omega_{0}, t\right)$ of Eq. (1). The corresponding generated monocycle waveform is plotted in Fig. 2(c). In this way, a positive and a negative pulse have been performed which are related with the channels at 1546.92 and $1547.72 \mathrm{~nm}$, respectively. According to the UWB classical waveforms definition [1], the obtained waveform corresponds to a monocycle IR-UWB pulse since both positive and negative lobes have the same amplitude, respect the $0 \mathrm{mV}$ of amplitude level. The generated electrical pulse is given by the incoherent optical signal power profile controlling both the polarity and the amplitude of the lobes of the generated waveform. Therefore, with the proper adjustment of the OCS attenuation of each channel, electrical IR-UWB pulses can be generated.

The electrical spectrum of the monocycle is shown in Fig. 2(d). Its central frequency is around $5 \mathrm{GHz}$ and the $10-\mathrm{dB}$ bandwidth is $7.25 \mathrm{GHz}$. In this way, the fractional bandwidth is $145 \%$ so UWB spectral definition is accomplished since it is larger than $20 \%$ of the pulse central frequency. As can be observed, baseband component is reduced corresponding to the generated electrical pulse in Fig. 2(c). This fact is a consequence of the appropriated selection of the incoherent optical signal power distribution using the OCS for a RF pulse generator pattern fixed. Therefore, the control of the incoherent optical signal power spectrum profile permits to improve the spectral efficiency of the generated UWB pulse.

\section{High ORDER UWB PULSES GENERATION}

In the following, the system flexibility for high order UWB pulses generation is experimentally demonstrated. In order to show this capability, different UWB waveforms have been generated performing specific shapings of the incoherent optical signal power distribution using the OCS.

First of all, a doublet pulse has been obtained. The incoherent optical signal spectrum is adjusted according to the power spectral distribution shown in Fig. 3(a) where three channels have been performed. In this case, the power of the two channels related to positive pulses is adjusted to be the half of a channel blocked. In this way, the equivalent amplitude levels correspond to [0.5, $-1,0.5]$. The resulting waveform is plotted in Fig. 3(b) which corresponds to a classical doublet pulse [1]. Fig. 3(c) depicts the electrical power spectrum of the doublet generated.

The flexibility of this system to increase the order of the pulses which can be obtained is demonstrated by generating a third and fourth order UWB pulses. This pulses are known as triplet and quadruplet, respectively. In the first case, four channels of the OCS are adjusted in a different way with equivalent amplitude levels [0.3, -1, 1, -0.3] performing the power profile distribution shown in Fig. 3(므). The obtained waveform is plotted in Fig. 3() $)$ which is according to the optical power profile and its corresponding electrical power spectra is depicted in Fig. 3(f). Finally, a quadruplet pulse has 
been implemented by modifying five channels of the OCS with equivalent amplitudes [-0.1, 0.6, -1, 0.6, -0.1]. In Figs 3(g), 3(h) and 3(i) are shown the power profile distribution, generated waveform and the corresponding electrical power spectrum, respectively. Therefore, the order of the UWB pulses generated can be easily increased by means of considering more channels of the OCS used.

For UWB indoor wireless communications, FCC establishes the higher power spectral restriction around the GPS band (0.96-1.61 GHz). As can be observed in Fig. 2(c), the monocycle electrical power spectrum is clearly not fulfilling the spectral requirements around GPS band. The level of the signal electrical power should be reduced in order to fulfill the FCC mask. In this way, the signal-to-noise ratio of the transmission system is sharply reduced driving to a restriction in the coverage distance [9]. Nevertheless, as can be observed in Figs. 3(c), 3(f) and 3(i), the generation of high order UWB pulses (doublet, triplet and quadruplet) permits to improve the fitting of the generated pulse spectra to the FCC mask. Therefore, an efficient solution in terms of power is achieved leading to an increase of the coverage distance.

\section{Adaptability to Bi-Phase Modulation}

In this section, we are going to show the capability of the system for being adapted to a Bi-Phase Modulation (BPM) transmission scheme. This corresponds to a binary modulation format in which each modulation state is given by a different signal polarity. In this sense, when the optical source is adjusted to have $[-1,1]$ as equivalent amplitude levels, the waveform shown in Fig. 4(a) is obtained. As can be observed, this corresponds to a inverted version of the generate monocycle pulse of Fig. 2(b). By the same procedure, the equivalent amplitudes can be adjusted to values such as $[-0.5$, $1,-0.5] ;[-0.3,1,-1,0.3]$ and $[0.1,-0.6,1,-0.6,0.1]$. The generated waveforms are shown in Figs. 4(b), 4(c) and 4(d) which correspond to the inverted version of the doublet, triplet and quadruplet of Figs. 3(b), 3(d) and 3(f), respectively.

Therefore, the full reconfigurable capability of the generated waveforms permits not only the synthesis of highorder IR-UWB pulses but the implementation of bi-phase modulation format.

\section{CONCLUSION}

In conclusion, we have proposed and experimentally demonstrated a technique for high-order UWB pulses generation based on the processing of an incoherent optical signal using a dispersive element. The use of incoherent optical sources permits to increase the flexibility in order to reconfigure the generated waveform which is given by the optical signal power profile. The proper control of the optical source power profile permits not only the generation of highorder pulses but also the reduction of the baseband component content. The feasibility of the system has been experimentally demonstrated by means of the generation of different waveforms: monocycle, doublet, triplet and quadruplet pulses. Furthermore, we have prove as the flexibility of the system permits to obtain the inverted version of the UWB pulses. This leads to the system adaptability to UWB transmission schemes in which BPM modulation format could be implemented.

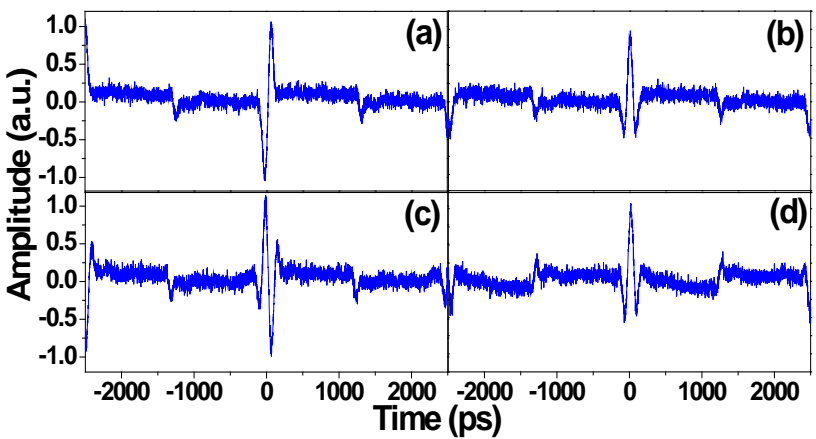

Fig. 4. Inverted pulses for (a) monocycle, (b) doublet, (c) triplet and (d) quadruplet.

\section{REFERENCES}

[1] L. Yang and G. B. Giammakis, "Ultra-Wideband communications: An idea whose time has come,” IEEE Signal Process. Mag., vol. 21, pp. 2654, November 2004.

[2] G. R. Aiello and G. D. Rogerson, "Ultra-Wideband wireless systems," IEEE Signal Process. Mag., vol. 4, pp. 36-47, June. 2003.

[3] J. Yao, "Photonics for Ultrawideband communications," IEEE Signal Process. Mag., vol. 10, pp. 82-95, June. 2009.

[4] T. B. Gibbon et al., "3.125 Gb/s Impulse Radio Ultra-Wideband Photonic Generation and Distribution Over a 50 km Fiber With Wireless Transmission," in IEEE Microwave and Wireless Components Letters, vol. 20, no. 2, pp. 127-129, Feb. 2010.

[5] J. Shao and J. Sun, "Photonic ultrawideband impulse radio shape modulation based on dual-filter tuning,” Opt. Lett., vol. 38, pp. 232-234, January 2013.

[6] K. Xu, X. Wu, J. Y. Sung, Z. Cheng, C. W. Chow, Q. H. Song and H. I. Tsang, "Amplitude and Phase Modulation of UWB Monocycle Pulses on a Silicon Photonic Chip,” IEEE Photon. Tech. Lett., vol. 28, pp. 248251, February 2016

[7] Y. Yu, J. Dong, X. Li and X. Zhang, "UWB monocycle generation and Bi-phase modulation based on Mach-Zehnder Modulator and Semiconductor Optical Amplifier,” IEEE Photon. J., vol. 4, pp. 327-339, April 2012.

[8] C. Wang and J. Yao, "All-fibre Ultrawideband Pulse generation based on Spectral Shaping and dispersión-induced Frequency-to-Time Mapping conversion,” IEEE Photon. Tech. Lett., vol. 19, pp. 137-139, February 2007.

[9] M. Bolea, J. Mora, B. Ortega and J. Capmany, “Optical UWB pulse generator using an $\mathrm{N}$ tap microwave photonic filter and phase inversion adaptable to different pulse modulation formats," Opt. Exp., vol. 17, pp. 5023-5032, March 2009.

[10] V. Moreno, M. Rius, J. Mora, M. A. Muriel and J. Capmany, "Integrable high order UWB pulse photonic generator based on cross-phase in a SOA-MZI," IEEE Microw. Mag., vol. 21, pp. 22911-22917, September 2013.

[11] P. Li, H. Chen, X. Wang, H. Yu, M. Chen and S. Xie, “Gigabit/s Photonic Generation, Modulation and Transmission for a Reconfigurable Impulse Radio UWB Over Fiber System” IEEE Photon. J., vol. 4, pp. 805-816, June 2012.

[12] V. Moreno, M. Rius, J. Mora, M. A. Muriel and J. Capmany, "Scalable UWB photonic generator based on the combination of doublet pulses," Opt. Exp., vol. 4, pp. 36-47, June 2014.

[13] Y. Park and J. Azaña, "Ultrahigh dispersion of broadband microwave signals by incoherent photonic processing," Opt. Exp., vol. 18, pp. 14752-14761, July 2010.

[14] M. Bolea, J. Mora, B. Ortega, J. Capmany, "Nonlinear dispersion-based incoherent photonic processing for microwave pulse generation with full reconfigurability," Opt. Exp, vol. 20, pp. 6728-6736, March 2014. 